Revista del Centro de Investigación de la Universidad La Salle

Vol. 12, No. 46, julio-diciembre, 2016: 39-78

http://ojs.dpi.ulsa.mx/index.php/rci/

\title{
Construcción de la EVIEES: Prácticas violentas por género en Educación \\ Superior ${ }^{1}$
}

\section{Construction of the EVIEES: Gender Violent Practices in Higher Education}

\author{
Leonor DELGADILLO-GUZMÁN \\ Francisco ARGÜELLO-ZEPEDA \\ Leonor GONZÁLEZ-VILLANUEVA
}

Fecha de recepción: 11 mayo de 2016

Fecha de aceptación: 12 enero de 2017

Disponible en línea: 23 marzo 2017

\section{Resumen}

Este trabajo tuvo como objetivo construir una escala psicométrica sobre violencia escolar entre estudiantes y estudiantes-profesores de educación pública superior para determinar las prácticas violentas por género, utilizando el modelo desarrollado por Delgadillo $(2010,2012)$. Es un estudio cuantitativo, transversal, exploratorio. Se trabajó con 744 estudiantes universitarios, 354 hombres, 388 mujeres; se obtuvieron valores estadísticos pertinentes: adecuación muestral KMO de .940; esfericidad de Bartlett de .000; valores de comunalidad superiores a .500; varianza total explicada de 44.15\%, 64 reactivos distribuidos en 8 factores; confiabilidad de $\alpha$ de Cronbach de .946; arrojando ocho indicadores: F1, física-psicológica; F2, desvalorización-control; F3, discriminación; F4, arbitrariedad; F5, indiferencia-desprecio; F6, desaprobación-rechazo; F7, abuso; F8, calumnia. Los resultados indican que las medias por grupo, según el sexo, los hombres

\footnotetext{
${ }^{1}$ E-mail: delgadilloleonor@gmail.com
}

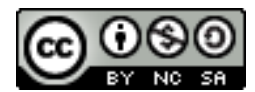

Revista del Centro de Investigación de la Universidad La Salle por Dirección de Posgrado e Investigación. Universidad La Salle Ciudad de México se distribuye bajo una Licencia Creative Commons Atribución-NoComercial-CompartirIgual 4.0 Internacional. 
Delgadillo, L.; Argüello, F.; González, L.

son quienes presentan significativamente más interacciones violentas y encabezan los registros de violencia a comparación de las mujeres, independientemente del nivel escolar. Disponer de una herramienta psicométrica técnicamente sólida es la mayor contribución de este trabajo, sobretodo hablando de un tema escasamente abordado, la violencia horizontal y vertical en educación púbica superior. Esto significa que las IES (Instituciones de Educación Superior) junto con el sector de la administración de justicia, podrán hacer uso de esta herramienta, EVIESS ${ }^{2}$ para documentar evidencia objetiva en caso de queja o denuncia de violencia escolar por pares o no pares, para la protección de la garantía establecida en el artículo $3^{\circ}$, fracción II, inciso “c” de la Constitución Política de los Estados Unidos Mexicanos.

Palabras clave: Violencia escolar, Educación superior, Género.

\footnotetext{
${ }^{2}$ Escala de Violencia en Estudiantes de Educación Superior, Delgadillo, Argüello y González, 2016.
} 


\section{Abstract}

The aim of this study is to build a psychometric scale about scholar violence between students and students-professors, who belong to public higher education to determine the violent practices by gender, using the Delgadillo $(2010,2012)$ proposal. This study is quantitative, transversal, and exploratory. It was worked with 744 college students, 354 men and 388 women; the results showed relevant statistical values: sampling adequacy KMO of .940; Bartlett sphericity of .000; values above .500 commonality; variance explained of $44.15 \%$, through 64 questions distributed into 8 factors; Cronbach $\alpha$ reliability of .946; they yielded eight indicators:, F1, physical-psychological; F2, underestimate-control; F3, discrimination; F4, injustice; F5, indifference-contempt; F6, disapproving-rejection; F7, abuse; F8, slander. The results showed that the mean per group according to sex, that men have significantly more violent interactions and lead records violence comparison of women, regardless of grade level. The main contribution of this paper is the construction of a specific psychometric for assessing de violence between peers and no peers, overall talking about a subject which is rarely speak it out, besides the initial interpretative of the data. This means that de Higher Education Institutions with the administration justice sector can use this scale for documenting objective evidence in case of suing or complaint because of scholar violence by peers and no peers for protecting the guarantee pointed in the third article, fraction II, subsection "c" of the Constitution of the United States of Mexico, both.

Key words: scholar violence, higher education, gender. 


\section{Introducción}

\section{Prácticas violentas en las escuelas}

La violencia escolar ha cobrado especial interés en los centros educativos durante los últimos años; profesores, padres de familia y autoridades educativas, han considerado la relación de intimidación, maltrato, o agresiones, como una preocupación de carácter social.

Las relaciones de dominio-sumisión que se presentan entre pares, donde la persona agresora busca hacer blanco en otra persona para hacerla su víctima produciéndole golpes, rechazos, insultos, con la finalidad de provocarle un daño físico ha incrementado en últimas fechas, sin embargo, conforme los grados escolares avanzan y la edad de los estudiantes aumenta, la violencia escolar aparentemente disminuye, o bien, sus formas se producen fundamentalmente de manera psicológica combinada, sobretodo, con la física o la sexual. Las investigaciones realizadas sobre violencia escolar se han enfocado con estudiantes de educación básica (primaria y secundaria) y de educación media superior; en el caso del nivel superior sólo se encontró el estudio realizado por de Delgadillo, Argüello, González y García (2012) sobre la situación que impera en México.

A nivel mundial se han realizado diversas investigaciones que reflejan la realidad del bullying. Olweus (1998) comenta que uno de cada siete alumnos en Noruega es víctima o participa en conductas de acoso. En investigaciones posteriores, Olweus (2003) estima que el 9\% de los estudiantes en las escuelas son víctimas o posibles objetivos de violencia escolar. Por su parte, Romans (2000) refiere que en España, un 40\% de los alumnos confiesan sentirse víctimas de la violencia en el contexto escolar. En este mismo país, del total de estudiantes de educación secundaria, el 50\% perciben problemas de agresión en las aulas y el 11\% de ellos, se sienten temerosos de otro estudiante (Del Barrio et al. 1999). En el mismo sentido, Ortega, Del Rey Sánchez, Ortega-Rivera y Genebat (2005) refieren que entre el 15 y 35\% del alumnado se ve involucrado en actitudes y comportamientos intimidatorios, abuso de poder y exclusión social.

En Canadá, Mac Donald (1999; citado por Velázquez, 2008) indica que el 31\% de los estudiantes 
de sexto a noveno grado habían sido víctimas de agresiones de sus compañeros, el 29\% había recibido amenazas y 16\% habían recibido golpes. En Francia, DeBarbieux y Blaya (2002) señalan que la prevalencia de maltrato entre escolares es cercana a 10\%. En Chile, Bellido (2010) identificó que el 10,7\% de los estudiantes se declaran víctimas de maltrato físico, amenazas y discriminación permanentes. Mientras que en Brasil, en una investigación desarrollada por Fisher (2010), con una muestra de 5,168 alumnos, el 28\% afirman haber sido víctimas de malos tratos por parte de sus colegas al menos una vez en el año 2009. En el mismo estudio, se documenta que el 10\% de ellos, comentan haber sufrido malos tratos, tres o más veces durante el mismo año.

En el caso de México, de los reportes publicados recientemente destaca el realizado por la Encuesta Nacional de Salud y Nutrición del 2006 (Olaiz, Rivera, Shamah, Rojas y Villalpando, 2006), el cual informa que prácticamente la cuarta parte de los participantes habían sido víctimas de bullying en algún momento, mientras que la Secretaría de Educación Pública (SEP) en colaboración con el Fondo para la Infancia de las Naciones Unidas (UNICEF, 2009) reportó que 43.2\% del personal docente sabía de la presencia de bullying en sus escuelas. Asimismo, un estudio realizado con más de un millón de estudiantes de secundaria, en diversas ciudades de Brasil y en el Distrito Federal, documentó que, del total, $83.4 \%$ había mencionado la presencia de bullying en su escuela (Abramovay, 2005). De la misma manera, en Guadalajara se reporta que hasta $68 \%$ de los alumnos de educación secundaria habían sido intimidados en algún momento (Valadez y Figueroa, 2008). Mientras que en Ciudad Juárez, Chihuahua, en una muestra de 1663 casos, 947 manifiestan la presencia de algún tipo de acoso entre compañeros; de estos, 174 casos corresponden al acoso físico, 437 al acoso verbal y 336 al acoso gesticular (Rodulfo, 2011).

Es importante comentar que la existencia de estudiantes que conjugan ser agresores y víctimas de acoso escolar representa una minoría, pues se estima que el 6\% de niños en Estados Unidos y Europa cumplen con dicho perfil (Nansel et al. 2004). 


\section{Antecedentes}

La violencia escolar ha llamado la atención desde finales de los años sesenta y principios de los setenta principalmente en Escandinavia, posteriormente en Japón, Inglaterra, Países Bajos, Canadá, Estados Unidos y Australia (Olweus, 2006, p. 17). El bullying es una forma de violencia escolar que se circunscribe al tipo de actores que se ven involucrados en ella, en este caso se trata exclusivamente de estudiantes (Del Rey y Ortega, 2001; Ortega y Del Rey, 2008). Sus manifestaciones pueden ser físicas, económicas, sexuales y psicológicas, ya sean específicas o combinadas (Delgadillo y Mercado, 2010, pp. 59-79).

Se ha encontrado que la incidencia y prevalencia de la violencia escolar entre pares, como se ha optado por llamarle en este estudio, varía dependiendo del país y de las medidas tomadas al respecto. Donde más se ha estudiado ha sido Escandinavia, Suecia, Escocia, Alemania, Francia, España, algunos países de América Latina ha iniciativa de universidades europeas con fines indagatorios y comparativos respecto a la situación de su país (Cerezo, 2001; Letamendia, 2002; Ortega, 2005; Krauskof, 2006; Del Rey y Ortega 2001, 2008; Ortega, Calmaestra y Mora 2001, 2008).

En México, Delgadillo et al. (2012) realizaron un estudio que tuvo por objetivo identificar el patrón de violencia escolar entre estudiantes de educación pública superior. Se trató de un estudio cualitativo (Ruiz, 1989). La hipótesis planteaba que el bullying producido entre estudiantes de educación pública superior es más sutil, sus expresiones son del orden de lo simbólico, una forma de violencia escolar psicológica, dadas las características psicosociales de los estudiantes, quienes a diferencia de los de educación básica cuentan con un mayor conocimiento social y procesos psicológicos de reflexión más acabados. Se trabajó con once estudiantes regulares de educación pública superior que se encontraban realizando sus estudios de licenciatura, seis varones y cinco mujeres.

Los hallazgos revelaron la existencia de la violencia en diferentes modalidades. Los autores indicaron que superar las práctica violentas resulta una utopía, ya que se observaron patrones 
escolares violentos, fundamentalmente la desvalorización y la indiferencia, formas psicológicas, la violencia sexual, y la violencia económica patrimonial, que se ven reforzadas dada la dinámica de exigencia académica que prevalece. Se espera de los estudiantes rasgos de personalidad pragmáticos e instrumentales, capaces de modificar el ambiente y la percepción de las personas, atributos propios de individuos con capacidad de liderazgo, lo que remite a una alta capacidad de influencia sobre el criterio de las personas, también con alta capacidad de logro, creativas, con alta habilidad en la comunicación. Este conjunto de características favorecen el individualismo y desalientan líneas de acción colaborativas y socialmente integradas. Los autores apuntan que, paradójicamente al fomentar el liderazgo, se estimula la integración social, sin embargo, habrá que tener presente que esta cualidad lleva implícita la competitividad, en consecuencia, una constante auto-referencialidad, lo que prevalece es el sí mismo, el egoísmo y no el agonismo (Delgadillo et al. 2012).

Los factores de riesgo en este tipo de población, y desde un sentido lato, se encontraría la baja o nula estética personal del individuo, así como, el nivel socioeconómico que se tenga, entre más bajo más posibilidades de sufrir violencia psicológica en el ámbito escolar. En contraste, como factores de protección, se observan líneas de acción que afrontan la violencia de manera activa o pasiva. Activa cuando se enfrenta a la persona agresora, cuestionando el porqué de su conducta, y pasiva cuando la forma de responder es mediante la evitación de contacto (Delgadillo et al. 2012). Pensar en este fenómeno y sus efectos nocivos, conduce a diversos cuestionamientos cómo explicar la reproducción de la violencia entre pares del mismo sexo, de diferente sexo, qué indicadores estadísticos se pueden construir para conocer su prevalencia, encarando con ello la reproducción de la desigualdad. Es así como es dable suponer que, al reproducirse la violencia entre pares estudiantes, intra e inter género de educación pública superior, se favorece la desigualdad y con ello la discriminación.

En virtud de lo anterior, queda en claro que el estudio de la violencia escolar representa un punto de agenda importante de investigación para las ciencias sociales, incluida la psicología, máxime 
cuando los registros de eventos violentos ilustran situaciones de alta peligrosidad. Dada esta situación y considerando la aportación que, desde la psicología puede verterse, se establecieron indicadores cuantitativos obtenidos a partir de una verificación teórico-metodológica que permitió evaluar las prácticas violentas entre estudiantes de educación pública superior, por medio del diseño de una escala para medir la frecuencia de violencia escolar entre ellos. Teniendo como beneficiarios de los resultados aquí obtenidos a las Universidades en general, y en lo particular a las Públicas.

\section{Modelo teórico}

Se trabajó con el modelo que sobre violencia ha desarrollado Delgadillo (et al. 2012; Delgadillo y Mercado, 2010) quien considera elementos conceptuales de la construcción social de la realidad (Schutz, 1993; Berger y Luckmann, 1978) que contempla la capacidad reflexiva, la intersubjetividad y significación de las experiencias vividas. El modelo de violencia mencionado atiende a las diferentes manifestaciones que puede tener la interacción dañina hacia otra(as) persona(as), ya se física, psicológica, económica o sexualmente.

\footnotetext{
"La realidad se construye socialmente y la sociología del conocimiento debe analizar los procesos por los cuales esto se produce...bastará con definir la «realidad» como una cualidad propia de los fenómenos que reconocemos como independientes de nuestra propia volición...y definir el «conocimiento» como la certidumbre de que los fenómenos son reales y de que poseen características específicas" (Berger y Luckmann, 1978, p. $13)$.
}

A partir de lo anterior, el modelo de Delgadillo (2010) propone un esquema tipológico sobre el patrón de actuación que puede seguir el receptor(a) de la violencia, con el que se ilustra que el receptor(a) puede resistirse o no a la violencia recibida y un segundo esquema tipológico sobre las formas de expresión de la violencia organizacional, que pueden tomar la forma franca o directa, o bien, la disfrazada, produciendo en sus relaciones distintas posibilidades de combinación (proyecto financiado por convocatoria por la UAEMéx, con clave 2291/2006). Ambos modelos son susceptibles de trasladarse al ámbito familiar y escolar. Estas elaboraciones conceptuales fueron la base para el desarrollo de la escala que aquí se presenta, así como otras que se han desarrollado sobre violencia docente (proyecto financiado por convocatoria por la Cámara de Diputados 
2756/2009) y en personal administrativo de educación superior (financiado por convocatoria por la UAEMéx, con clave 2597/2008).

De manera esquemática la elaboración teórica del instrumento piloto fue la siguiente:

\section{Imagen 1.}

Esquema tipológico del patrón de actuación desde el receptor(a) de la violencia

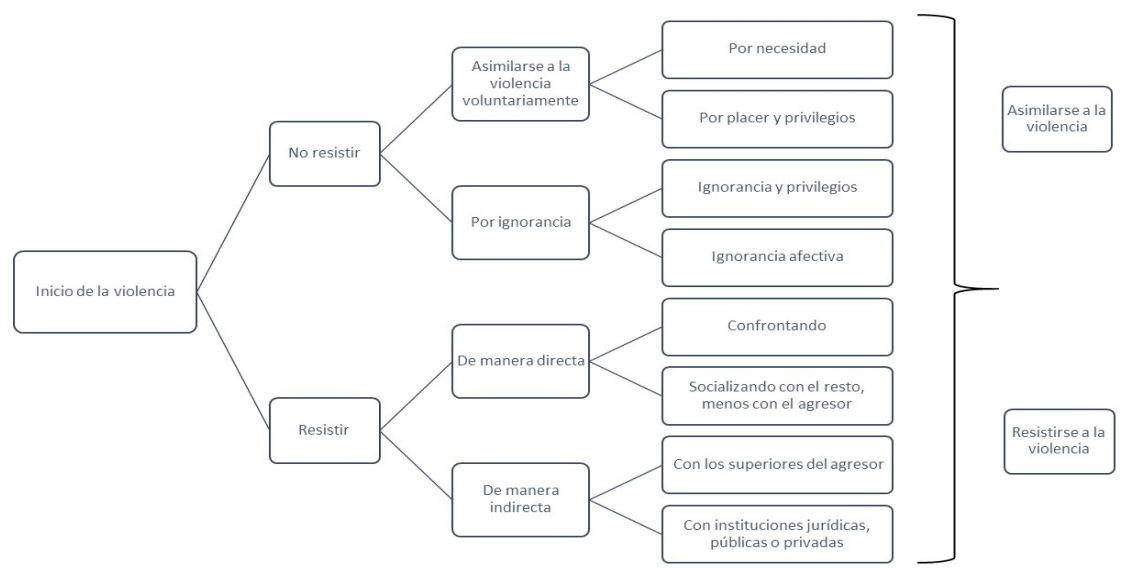

Retomado de Delgadillo (2010) 


\section{Imagen 2.}

\section{Esquema tipológico sobre las formas de expresión de la violencia organizacional}

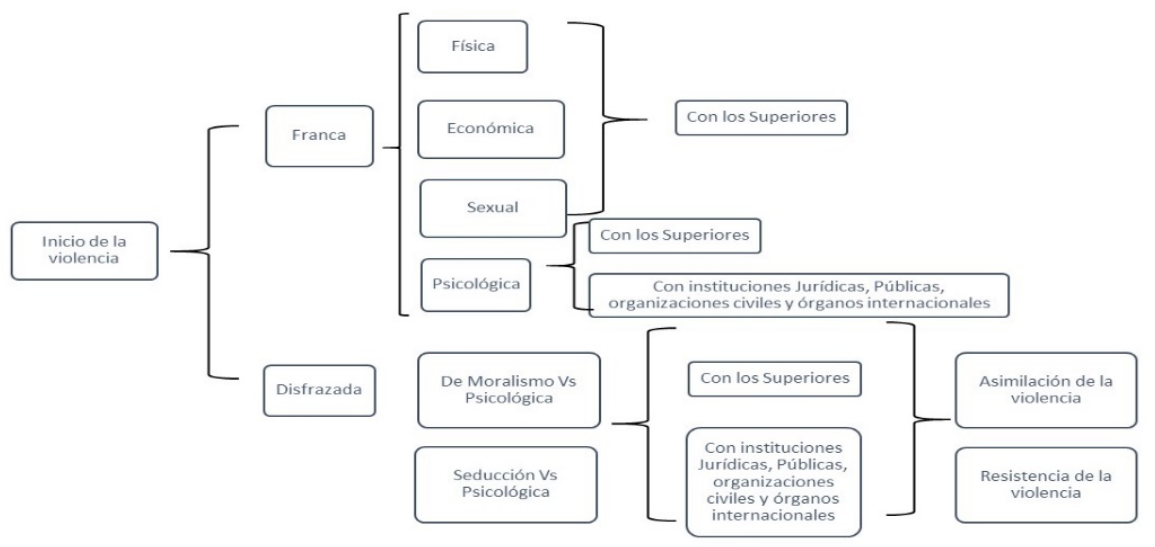

Retomado de Delgadillo (2010)

Los hechos violentos que involucran a los estudiantes se han presentado en diversas partes del mundo, sin tener certeza de qué les lleva a presentar este tipo de conductas; se tiene una compleja red de factores biológicos, psicológicos y sociales que en conjunto pueden dar como resultado un individuo violento.

\section{Método}

\section{Pregunta de investigación}

¿Cuáles son los indicadores sobre prácticas violentas que se reportarán los estudiantes de educación pública superior?

\section{Objetivo General}

Construir una escala psicométrica sobre violencia escolar entre estudiantes y estudiantesprofesores de educación pública superior para determinar las prácticas violentas por género.

\section{Objetivos especificos}

Establecer indicadores cuantitativos obtenidos a partir de una verificación teórico-metodológica que permitan evaluar las prácticas violentas entre estudiantes de educación pública superior. 
Identificar la frecuencia de las prácticas violentas entre estudiantes de educación pública superior. Determinar las prácticas violentas verticales entre estudiantes y profesores de educación pública superior.

Determinar las prácticas violentas verticales entre estudiantes y profesores de educación pública superior.

Identificar las prácticas violentas por sexo entre estudiantes de educación pública superior.

\section{Hipótesis}

La EVIEES será una escala sólida con validez y confiabilidad necesaria para determinar las prácticas violentas en educación pública superior.

Existirán prácticas violentas horizontales entre estudiantes de educación pública superior.

Existirán prácticas violentas verticales entre estudiantes y profesores de educación pública superior.

Existirán diferencias significativas de las prácticas violentas horizontales y verticales por género.

\section{Planteamiento del problema}

La psicología como ciencia de investigación, pretende estudiar a las personas en sociedad, la situación actual exige un estudio, análisis y comprensión de los fenómenos que se presentan para poder dar alternativas al respecto. Una de las aportaciones ha sido la construcción de instrumentos de medición que permitan conocer al ser humano con mayor objetividad. En base a ello, se observó la necesidad de elaborar un instrumento que permite determinar los tipos de prácticas violentas entre estudiantes; así mismo, entre estudiantes y profesores de educación pública superior, así como su frecuencia.

La presente, surge como un producto del proyecto de investigación "Prácticas violentas por género en estudios de educación pública superiores", financiado por la Universidad Autónoma del Estado de México (registrado con clave 3440-2013CHT), con la finalidad de conocer las relaciones violentas de los universitarios, con el fin de conocer la situación del panorama universitario y la 
Delgadillo, L.; Argüello, F.; González, L.

realidad que enfrentan algunos grupos vulnerables.

Variable de estudio

Violencia escolar (ver tabla 1): el elemento teórico básico que reúne el fenómeno de estudio violencia escolar- es entendido como una expresión negativa del poder, consiste en todo acto dañino intencional (voluntario) emitido por un estudiante de la escuela, que puede producirse a través de la acción u omisión, acto que va dirigido en contra de otra(s) persona(s), estudiantes de la escuela, cuya intención es dominarle tendiendo hacia la eliminación del compañero de la escuela, o al abuso del compañero y llegando incluso a su eliminación (homicidio). Estos actos pueden ser físicos, económicos, sexuales o psicológicos, pudiendo manifestarse de manera directa o inversa. Generalmente se trata de actos que se presentan de manera combinada y tienen repercusiones en la salud del receptor (Delgadillo y Mercado, 2010). 
Tabla 1

Operacionalización de la escala

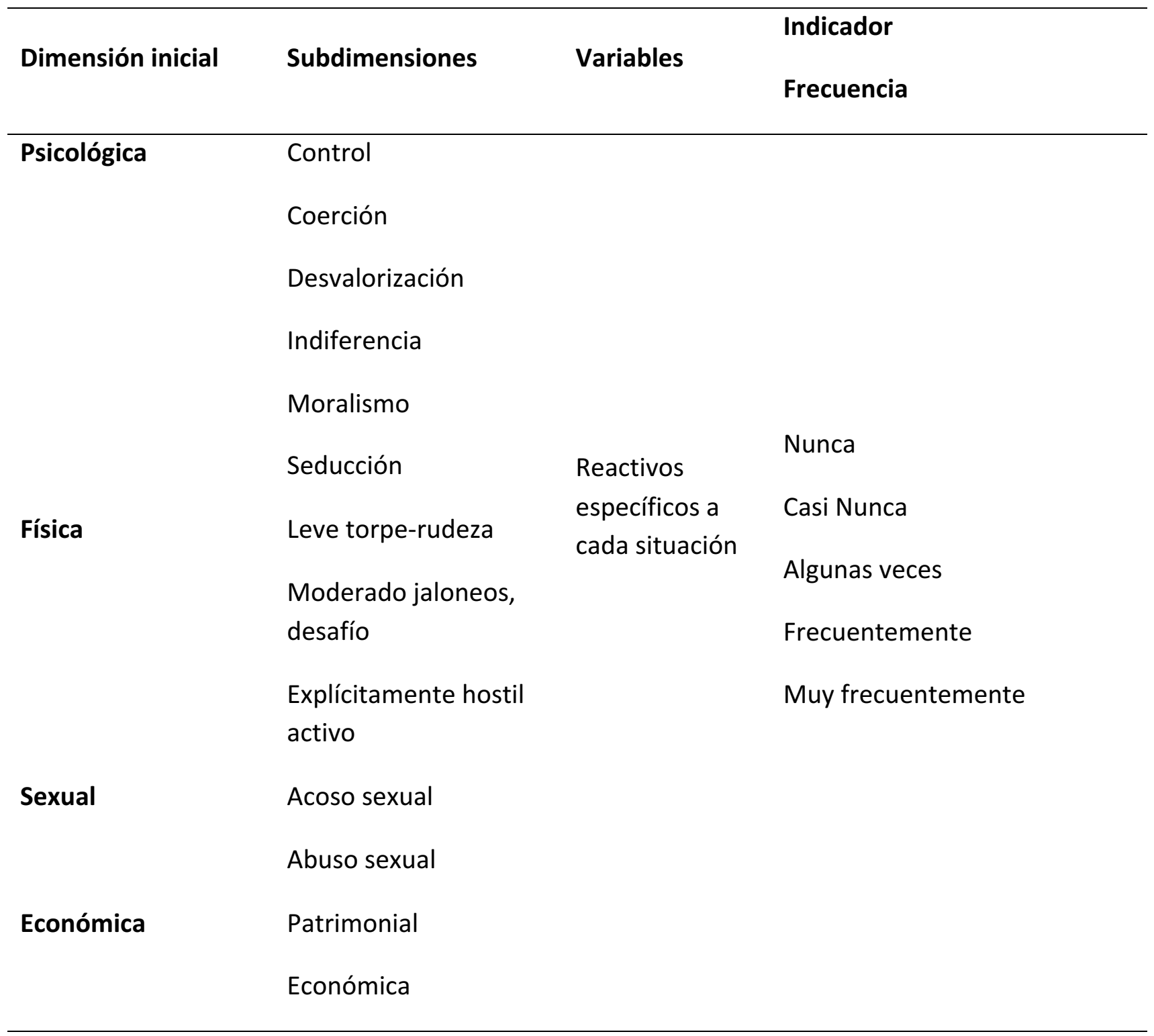




\section{Muestra}

Se trabajó 744 participantes estudiantes de educación pública superior, cursando del 3ro al 11vo semestre de 25 licenciaturas de la Universidad Autónoma del Estado de México, siendo: 354 hombres y 388 mujeres; con edad mínima de 18 años y máximo de 35 años, con una media de 22 años; por estado civil: 706 solteros, 21 casados, y 16 en unión libre; 36 tienen hijos; 192 trabajan y estudian; 543 profesan religión católica, 42 cristiana, 3 judía y 2 testigos de Jehovah y 143 no son practicantes; 34 poseen algún tipo de discapacidad, principalmente de tipo visual; 35 tienen alguna enfermedad crónica, principalmente rinitis-alérgica, asma y miopía; 36 pertenecen a algún grupo indígena: 15 otomís, 20 mazahuas y 1 matlatzinca; la mayoría viven con sus padres y hermanos; y también, la mayoría tienen el primer lugar entre sus hermanos.

\section{Procedimiento}

Se diseñó la escala con base al modelo teórico y estudios empíricos previos, considerando violencia vertical de docente hacia estudiante, y violencia horizontal de estudiante a estudiante; se procedió al jueceo de expertos para la modificación teórico-técnica de reactivos; se aplicó una prueba piloto con 108 reactivos; se corrigió la escala; se procedió a la aplicación de campo en la muestra de estudio integrando el consentimiento informado, anonimato y confidencialidad estricta de los sujetos; se integró la base de datos en paquete estadístico, SPSS; se revisó la adecuación muestral para el análisis factorial con las pruebas de KMO y prueba de Bartlett; se obtuvo el índice de validez a través del análisis factorial y gráfico de sedimentación factorial; la varianza explicada a través de los valores Eigen; los factores de la escala; el índice de confiabilidad por el $\alpha$ de Cronbach, método que se basa en el análisis de las inter-correlaciones promedios entre los reactivos a partir de una aplicación de la prueba (Thorndike, 1989).

Lo anterior, condujo a la integración de la versión final de la escala de acuerdo a los criterios psicométricos obtenidos (ver anexo 1). Análisis comparativo de las prácticas violentas por género a través de la prueba estadística t de student, análisis comparativos de las prácticas violentas horizontales y verticales a través del estudio de medias y $\mathrm{t}$ de student. 


\section{Resultados}

En general, los resultados obtenidos arrojaron valores pertinentes; primero, la medida de adecuación muestral de Kaise-Meyer-Olkin, que fue de .940; y de la correlación obtenida de las variables, siguiendo el criterio de la esfericidad de Bartlett de .000 (ver tabla 2), se obtuvieron los valores del criterio de comunalidad, mismos que arrojaron registros superiores a .500

\section{Tabla 2}

KMO y prueba de Bartlett

\begin{tabular}{l}
\hline Medida de adecuación muestral de Kaiser- \\
Meyer-Olkin.
\end{tabular}

\begin{tabular}{llc}
$\begin{array}{ll}\text { Prueba de esfericidad } \\
\text { de Bartlett }\end{array}$ & $\begin{array}{l}\text { Chi-cuadrado } \\
\text { aproximado }\end{array}$ & 3996E4 \\
& gl & 5778 \\
& Sig. & .000 \\
\hline
\end{tabular}

La varianza explicada obtenida en este instrumento fue de $44.15 \%$, explicando en 8 factores (ver tabla 3) que aglutinan un total de 64 reactivos. La confiabilidad obtenida fue de $\alpha$ de Cronbach .946. Hablando en términos descriptivos sobre los factores, a continuación, se presentan cada uno de ellos junto con la caracterización de los reactivos que los configura (ver tabla 4). 
Delgadillo, L.; Argüello, F.; González, L.

\section{Tabla 3}

Varianza total explicada

Componente Autovalores iniciales

Sumas de las saturaciones Suma de las saturaciones

al cuadrado de la al cuadrado de la rotación

extracción

\begin{tabular}{|c|c|c|c|c|c|c|c|c|c|}
\hline \multirow[b]{3}{*}{1} & \multirow{3}{*}{$\begin{array}{l}\text { Total } \\
22.804\end{array}$} & \multirow{2}{*}{\multicolumn{2}{|c|}{$\begin{array}{l}\% \text { de la } \% \\
\text { varianzaacumulad }\end{array}$}} & \multirow{2}{*}{ Total } & \multirow{2}{*}{\multicolumn{3}{|c|}{$\begin{array}{l}\text { \% de la } \% \quad \text { Total } \\
\text { varianzaacumulado }\end{array}$}} & \multirow{2}{*}{\multicolumn{2}{|c|}{$\begin{array}{l}\% \text { de la } \% \\
\text { varianzaacumulado }\end{array}$}} \\
\hline & & & & & & & & & \\
\hline & & 21.115 & 21.115 & 22.804 & 421.115 & 21.115 & 8.473 & 7.845 & 7.845 \\
\hline 2 & 9.863 & 9.132 & 30.247 & 9.863 & 9.132 & 30.247 & 6.982 & 6.465 & 14.310 \\
\hline 3 & 4.197 & 3.886 & 34.134 & 4.197 & 3.886 & 34.134 & 5.120 & 4.741 & 19.051 \\
\hline 4 & 3.073 & 2.846 & 36.979 & 3.073 & 2.846 & 36.979 & 5.006 & 4.635 & 23.686 \\
\hline 5 & 2.178 & 2.017 & 38.996 & 2.178 & 2.017 & 38.996 & 4.300 & 3.982 & 27.668 \\
\hline 6 & 2.019 & 1.869 & 40.865 & 2.019 & 1.869 & 40.865 & 3.873 & 3.586 & 31.254 \\
\hline 7 & 1.824 & 1.689 & 42.554 & 1.824 & 1.689 & 42.554 & 2.881 & 2.668 & 33.922 \\
\hline 8 & 1.728 & 1.600 & 44.154 & 1.728 & 1.600 & 44.154 & 2.817 & 2.608 & 36.530 \\
\hline 9 & 1.676 & 1.552 & 45.706 & 1.676 & 1.552 & 45.706 & 2.762 & 2.557 & 39.088 \\
\hline 10 & 1.581 & 1.464 & 47.171 & 1.581 & 1.464 & 47.171 & 2.292 & 2.122 & 41.210 \\
\hline 11 & 1.498 & 1.387 & 48.558 & 1.498 & 1.387 & 48.558 & 2.271 & 2.103 & 43.313 \\
\hline
\end{tabular}

El Factor 1, violencia horizontal física-psicológica, compuesto por 16 reactivos: se golpean, se insultan, se dicen palabras obscenas, se gritan, se callan groseramente, se hablan mal entre sí, se llevan de manera tosca, se ofenden, se arrebatan las cosas, se saludan de "bulto" (con golpes, empujones), se lanzan objetos, se amenazan entre sí, cuando pasan junto al otro se empujan sin motivo, agreden a otros sin causa aparente, se humillan, se hacen burlas públicas. 
Factor 2, violencia vertical desvalorización-control de profesores a estudiantes, compuesto por 11 reactivos: a través de intimidación logran hacer que un alumno esté en contra de otro, rechazan aquellos(as) que tienen rasgos indígenas, intimidan al alumno para que realice "algo" para ellos, insta al alumno a realizar actividades para burlarse de él, dan menos valor al trabajo del estudiante discapacitado, presentan los trabajos de los alumnos como propios, rechazan al alumno que es homosexual, se valen del alumno(a) para que realice trabajos extras de su conveniencia, utilizan el chantaje como beneficio personal, condicionan la calificación a un trato personal; favorecen al alumno(a) con permisos especiales que después se cobran.

Factor 3, violencia vertical discriminación, compuesto por ocho reactivos: tratan bien a los que son "guapos" o "guapas"; los(as) carismáticos(as) son sus preferidos(das), los guapos(as) son sus preferidos(as), rechazan a $\operatorname{los}(\mathrm{as})$ feos(as), favorecen a los que visten bien, tiene a sus preferidos(as), son indiferentes con los alumnos(as) menos "listos(as)", relegan a los(as) estudiantes que no les simpatizan.

Factor 4, violencia vertical arbitrariedad, compuesto por ocho reactivos: cambian el esquema de evaluación sin previo aviso, cambian los lineamientos de la entrega de trabajos sin previo aviso, hacen esperar a los estudiantes; dan órdenes abusando de su autoridad, abusan de su autoridad, sancionan al alumno(a) sin escuchar razones o circunstancias, regañan denigrando a los estudiantes, se dirigen a los alumnos(as) con groserías elevando el tono de voz.

Factor 5, violencia horizontal indiferencia-desprecio, con siete reactivos: se dejan de hablar sin motivo aparente, aplican la ley del hielo, se ignoran como una forma de agredirse, utilizan el chantaje para beneficio personal, se miran "feo", se dejan con "la palabra en la boca", se rechazan evitando sentarse junto a un compañero(a).

Factor 6, violencia horizontal desaprobación-rechazo, con siete reactivos: se critican por la ropa que usan; se critican la forma de vestir, se critican por su forma de ser, hablan mal a sus espaldas 
Delgadillo, L.; Argüello, F.; González, L.

sin razón, se insultan por ser de diferente clase social, ignoran a compañeros(as) al integrar equipos, se excluyen de trabajos por no caerse bien.

Factor 7, violencia vertical abuso, con cuatro reactivos: muestran indiferencia a los alumnos(as) indisciplinados(as), impiden el acceso al salón al alumno(a) que no le simpatiza por rebasar el tiempo de tolerancia, deja una mayor carga de trabajo superior a la indicada en el programa, condicionan la calificación contra-entrega de algún tipo de material.

Factor 8, violencia horizontal calumnia, con tres reactivos: esparcen rumores para desacreditar a un(a) compañero(a), se crean chismes divulgándolos entre ellos, se desacreditan con chismes. 
Tabla 4

Cargas factoriales por indicador

Contenido del Reactivo

\section{Factores}

\begin{tabular}{llllllll}
\hline 1 & 2 & 3 & 4 & 5 & 6 & 7 & 8
\end{tabular}

46. Se golpean.

.705

33. Se insultan.

.701

49. Se dicen palabras obscenas.

.699

19. Se gritan.

.660

17. Se callan groseramente.

.637

03. Se hablan mal entre sí.

.617

14. Se llevan de manera tosca.

09. Se ofenden.

26. Se arrebatan las cosas. .602

37. Se saludan de "bulto" (con golpes, empujones). .595

60. Se lanzan objetos.

08. Se amenazan entre sí.

54. - Cuando pasan junto al otro(a) se empujan sin motivo.

02. Agreden a otros sin causa aparente. 
23. Se hacen burlas públicas.

93. A través de intimidación logran hacer que un alumno(a) esté contra de otro(a).

96. Rechazan a los (las) que tienen rasgos indígenas.

83. Intimidan al alumno(a) para que realice "algo" para ellos(as).

82. Instan al alumno(a) a realizar actividades para burlarse de él(la).

81. Dan menos valor al trabajo del estudiante discapacitado (a).

91. Presentan los trabajos de los alumnos (as) como propios.

90. Rechazan al alumno (a) que es homosexual.

107. Se valen del alumno(a) para que realice trabajos extras de su conveniencia.

95. Utilizan el chantaje como beneficio personal.

99. Condicionan la calificación a un trato personal.

106. Favorecen al alumno(a) con permisos especiales que después se cobran.

103. Tratan bien a los que son guapos o guapas.

100. Los(as) carismáticos(as) son sus preferidos(as).

73. Los guapos(as) son sus preferidos(as).

.688

89. Rechazan a los(as) feos(as).

.616

87. Favorecen a los que visten bien.

.593

86. Tienen a sus preferidos(as).

.572 
88. Son indiferentes con los alumnos (as) menos "listos" (as).

80. Relegan a los(as) estudiantes que no les simpatizan.

97. Cambian el esquema de evaluación sin previo aviso.

85. Cambian los lineamientos de la entrega de trabajos sin previo aviso.

94. Hacen esperar a los estudiantes.

84. Dan órdenes abusando de su autoridad.

78. Abusan de su autoridad.

101. Sancionan al alumno(a) sin escuchar razones o circunstancias.

98. Regañan denigrando a los estudiantes.

108. Algunos profesores se dirigen a los alumnos con groserías, elevando el tono de voz.

21. Se dejan de hablar sin motivo aparente.

53. Aplican la ley del hielo.

24. Se ignoran como una forma de agredirse.

22. Utilizan el chantaje para beneficio personal.

39. Se miran feo.

44. Se dejan con "la palabra en la boca".

50. Se rechazan evitando sentarse junto a un compañero (a). 
Delgadillo, L.; Argüello, F.; González, L.

36. Se critican la forma de vestir.

11. Se critican por su forma de ser.

31. Hablan mal a sus espaldas sin razón.

61. Se insultan por ser de diferente clase social.

35. Ignoran a compañeros(as) al integrar equipos.

57. Se excluyen de trabajos por no caerse bien.

62. Muestran indiferencia a los alumnos (as) indisciplinados (as).

43. Impiden el acceso al salón al alumno(a) que no le simpatiza por rebasar el tiempo de tolerancia.

63. Dejan una mayor carga de trabajo, superior a la indicada en el programa.

64. Condicionan la calificación contra-entrega de algún tipo de material

05. Esparcen rumores para desacreditar a un(a) compañero (a).

15. Se crean chismes divulgándolos entre ellos.

25. Se desacreditan con chismes.

Se obtuvieron las medias por grupo de acuerdo al sexo (ver tabla 5) y se observó que los hombres presentan interacciones violentas con mayor frecuencia que las mujeres, con una diferencia estadísticamente significativa, estos datos revelan que independientemente del nivel escolar, el sexo masculino continúa encabezando los registros de violencia en comparación con el sexo femenino (ver tabla 6) 


\section{Tabla 5}

Prueba t entre grupos por sexo

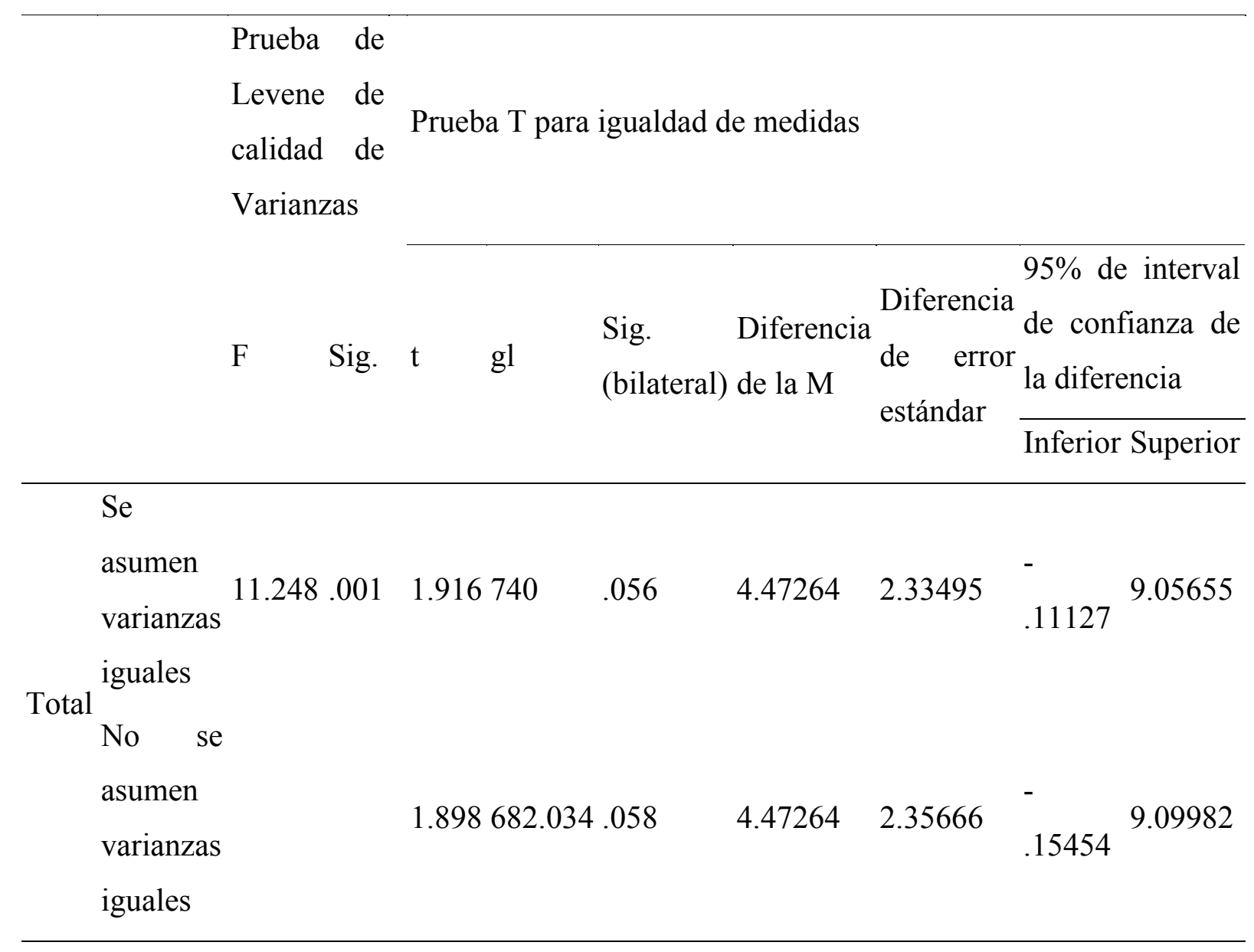




\section{Tabla 6}

Medias entre grupos por sexo

\begin{tabular}{lllll}
\hline Sexo & $\boldsymbol{n}$ & $\boldsymbol{M}$ & $\boldsymbol{D E}$ & $\begin{array}{l}\text { Diferencia de } \\
\text { error } \\
\text { estándar }\end{array}$ \\
\hline Hombre & 354 & 1.43 & 34.99 & 1.85 \\
Mujer & 388 & 1.38 & 28.52 & 1.48 \\
\hline
\end{tabular}

Al obtener el valor del modo por factor se observa que la frecuencia máxima en cinco de los ocho factores fluctúa del "casi nunca" al "algunas veces", factor 1, "Violencia horizontal físicapsicológica"; factor cuatro, "Violencia vertical arbitrariedad"; factor cinco, "Violencia horizontal indiferencia-desprecio"; factor seis, "Violencia horizontal desaprobación-rechazo"; y factor ocho, "Violencia horizontal calumnia"; menos en los factores dos, "Violencia vertical desvalorizacióncontrol"; factor tres, "Violencia vertical discriminación"; factor siete, "Violencia vertical abuso" de las prácticas violentas entre estudiantes de educación pública superior. Tal y como se observa en la tabla número 7.

\section{Tabla 7}

\section{Estadísticos de media y moda}

\begin{tabular}{lllllllll}
\hline Factor & F1 & F2 & F3 & F4 & F5 & F6 & F7 & F8 \\
n válido & 11888 & 8173 & 5944 & 5944 & 5201 & 5201 & 2972 & 2229 \\
Perdidos & 0 & 3715 & 5944 & 5944 & 6687 & 6687 & 8916 & 9659 \\
Media & 2.4407 & 1.5461 & 2.2246 & 2.3124 & 2.2525 & 2.4391 & 2.2365 & 2.1857 \\
Rango & $1-5$ & & & & & & & \\
Moda & 2.00 & \multirow{2}{*}{1.00} & \multirow{2}{*}{1.00} & 2.00 & 2.00 & 2.00 & 1.00 & 2.00 \\
\hline
\end{tabular}

Estos datos reflejan aparentemente, en lo general, una baja repetición de las interacciones significativamente violentas actuales en el ambiente académico de los estudiantes de forma horizontal y vertical. Se puede pensar al respecto que ello obedece por un lado a una tolerancia de 
la violencia tomándola como algo natural, negando su persistencia al punto de minimizarla y, por otro lado, la conformación de grupos diversos entre estudiantes cuyas prácticas concretas de interacción, efectivamente se presentan de forma escasa o intermitente. No obstante, en nuestra opinión, se tendría que estar alerta de su manifestación para monitorear el patrón que muestra de tiempo en tiempo, por lo menos una vez al año en los centros académicos.

Al identificar las prácticas violentas por sexo entre estudiantes de educación pública superior, se advierte una diferencia entre medias en todos los factores. Los factores en los que los varones

presentaron mayor interacción violenta fueron: factor uno, "Violencia horizontal físicapsicológica"; factor dos, "Violencia vertical desvalorización-control"; factor siete, "Violencia vertical abuso". Los factores en los que las mujeres presentaron mayor interacción violenta fueron: factor tres, "Violencia vertical discriminación"; factor cuatro, "Violencia vertical arbitrariedad"; factor cinco, "Violencia horizontal indiferencia-desprecio"; factor seis, "Violencia horizontal desaprobación-rechazo"; factor ocho, "Violencia horizontal calumnia" (ver tabla 8). 
Delgadillo, L.; Argüello, F.; González, L.

\section{Tabla 8}

Estadísticos por grupo

\begin{tabular}{|c|c|c|c|c|c|}
\hline Factor & Sexo & n & Media & DE & $\begin{array}{l}\text { Diferencia de } \\
\text { error } \\
\text { estándar }\end{array}$ \\
\hline \multirow[t]{2}{*}{ F1 } & Hombre & 354 & 41.9237 & 11.86813 & .63078 \\
\hline & Mujer & 388 & 36.4304 & 10.14611 & .51509 \\
\hline \multirow[t]{2}{*}{ F2 } & Hombre & 354 & 17.6073 & 6.63163 & .35247 \\
\hline & Mujer & 388 & 16.4485 & 5.12076 & .25997 \\
\hline \multirow[t]{2}{*}{ F3 } & Hombre & 354 & 17.4972 & 6.49046 & .34496 \\
\hline & Mujer & 388 & 18.0670 & 6.18790 & .31414 \\
\hline \multirow[t]{2}{*}{ F4 } & Hombre & 354 & 18.3192 & 6.11526 & .32502 \\
\hline & Mujer & 388 & 18.6546 & 5.99305 & .30425 \\
\hline \multirow[t]{2}{*}{ F5 } & Hombre & 354 & 15.3870 & 5.53177 & .29401 \\
\hline & Mujer & 388 & 16.1057 & 4.75896 & .24160 \\
\hline \multirow[t]{2}{*}{ F6 } & Hombre & 354 & 16.4407 & 5.70702 & .30332 \\
\hline & Mujer & 388 & 17.6443 & 5.61120 & .28487 \\
\hline \multirow[t]{2}{*}{ F7 } & Hombre & 354 & 9.3390 & 3.33890 & .17746 \\
\hline & Mujer & 388 & 8.5799 & 2.69487 & .13681 \\
\hline \multirow[t]{2}{*}{ F8 } & Hombre & 354 & 6.4972 & 2.91450 & .15490 \\
\hline & Mujer & 388 & 6.6082 & 2.69963 & .13705 \\
\hline
\end{tabular}


Los resultados observados apuntan a conjuntos de prácticas violentas diferenciados por sexo, aseveración que fue sometida a análisis estadístico que mostró cuatro factores con diferencias significativas, factor uno, "Violencia horizontal física-psicológica", los varones muestran mayor violencia que las mujeres; factor dos, "Violencia vertical desvalorización-control”, igualmente los varones presentan mayor violencia que las mujeres; factor cinco, "Violencia horizontal indiferencia-rechazo", en este factor las mujeres presentan mayor violencia que los varones; factor siete, violencia vertical abuso", una vez más los varones presentan mayor violencia que las mujeres (ver tabla 9). 
Delgadillo, L.; Argüello, F.; González, L.

Tabla 9

Pruebas T por factor

\begin{tabular}{|c|c|c|c|c|c|c|c|}
\hline \multirow{2}{*}{ Factor } & \multicolumn{2}{|c|}{$\begin{array}{l}\text { Prueba de Levene } \\
\text { de calidad de } \\
\text { Varianzas }\end{array}$} & \multirow[b]{2}{*}{$t$} & \multirow[b]{2}{*}{$g l$} & \multirow[b]{2}{*}{$\begin{array}{l}\text { Sig. } \\
\text { (bilateral) }\end{array}$} & \multirow[b]{2}{*}{$\begin{array}{l}\text { Diferencia de } \\
\text { la M }\end{array}$} & \multirow[b]{2}{*}{$\begin{array}{l}\text { Diferencia de } \\
\text { error estándar }\end{array}$} \\
\hline & $\boldsymbol{F}$ & Sig. & & & & & \\
\hline \multirow[t]{2}{*}{ F1 } & 8.021 & .005 & 6.794 & 740 & .000 & 5.49332 & .80859 \\
\hline & & & 6.745 & 697.742 & .000 & 5.49332 & .81438 \\
\hline \multirow[t]{2}{*}{$\mathrm{F} 2$} & 19.934 & .000 & 2.677 & 740 & .008 & 1.15889 & .43291 \\
\hline & & & 2.646 & 662.655 & .008 & 1.15889 & .43797 \\
\hline \multirow[t]{2}{*}{ F3 } & .001 & .978 & -1.224 & 740 & .221 & -.56984 & .46555 \\
\hline & & & -1.221 & 725.892 & .222 & -.56984 & .46657 \\
\hline \multirow[t]{2}{*}{ F4 } & .103 & .748 & -.754 & 740 & .451 & -.33543 & .44479 \\
\hline & & & -.753 & 730.831 & .451 & -.33543 & .44521 \\
\hline \multirow[t]{2}{*}{ F5 } & 8.399 & .004 & -1.902 & 740 & .058 & -.71866 & .37794 \\
\hline & & & -1.889 & 699.682 & .059 & -.71866 & .38054 \\
\hline \multirow[t]{2}{*}{ F6 } & .081 & .776 & -2.895 & 740 & .004 & -1.20365 & .41580 \\
\hline & & & -2.893 & 731.348 & .004 & -1.20365 & .41612 \\
\hline \multirow[t]{2}{*}{ F7 } & 11.194 & .001 & 3.421 & 740 & .001 & .75909 & .22192 \\
\hline & & & 3.388 & 678.640 & .001 & .75909 & .22407 \\
\hline
\end{tabular}


F8

1.806

$.179-.539740$

.590

$-.11107$

.20611

$-.537719 .698$

.591

$-.11107$

.20683

Los análisis arrojan la existencia de prácticas violentas, tanto vertical, como horizontal, existiendo diferencias estadísticamente significativas que presentan una mayor prevalencia de violencia entre alumnos, que la violencia entre alumnos y profesores. A continuación, se detallan los resultados (tabla 10 y tabla 11).

\section{Tabla 10}

Medias entre violencia horizontal y violencia vertical

\begin{tabular}{|c|c|c|c|c|c|}
\hline $\begin{array}{l}\text { Tipo de } \\
\text { violenci } N \\
\text { a }\end{array}$ & $M$ & $D E$ & $\begin{array}{l}\text { n } \\
\text { reactivos }\end{array}$ & de ${ }^{\text {rango }}$ & $\begin{array}{l}\text { M de error } \\
\text { estándar }\end{array}$ \\
\hline $\begin{array}{l}\text { Horizont } \\
\text { al }\end{array}$ & 76 & 20.48 & 60 & $60-300$ & .75 \\
\hline Vertical 743 & 60 & 17.92 & 66 & $66-330$ & .66 \\
\hline
\end{tabular}




\section{Tabla 11}

Prueba t entre tipos de violencia

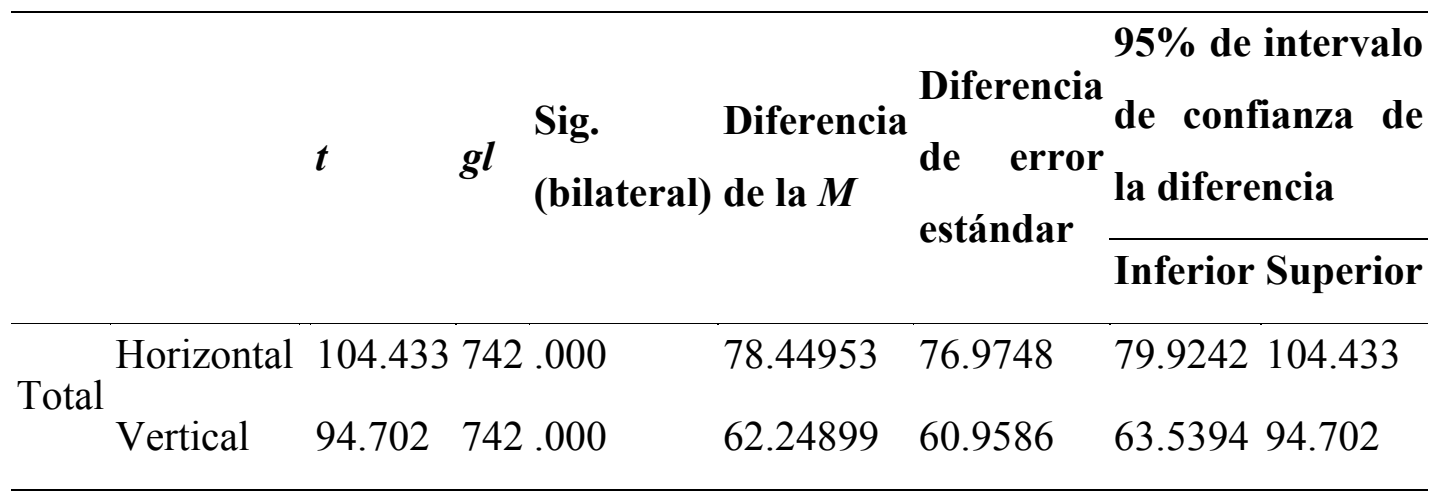

\section{Discusión y conclusiones}

Respecto a la primera hipótesis, el modelo teórico-metodológico representa un esquema adecuado para la construcción de herramientas psicométricas en materia de violencia escolar (Delgadillo, 2012; Delgadillo y Mercado, 2010). El instrumento construido, Escala de Violencia de Estudiantes de Educación Superior (EVIEES) presenta valores pertinentes de construcción, la medida de adecuación muestral de Kaise-Meyer-Olkin fue de .940; la correlación de las variables, siguiendo el criterio de la esfericidad de Bartlett, fue de .000. Los valores de comunalidad arrojaron registros superiores a .500. La varianza explicada fue de 44.15\%. La confiabilidad obtenida fue de $\alpha$ de Cronbach .946, es decir, es una escala sólida con validez y confiabilidad necesarios para determinar las prácticas violentas en educación pública superior.

La EVIEES (Delgadillo, Argüello y González, 2016) representa una escala sólida con validez y confiabilidad necesarios para determinar las prácticas violentas en educación pública superior. Se obtuvieron indicadores cuantitativos puntuales para evaluar las prácticas violentas entre estudiantes de educación pública superior, a partir de la propuesta teórica-metodológica desarrollada: Factor 1, violencia horizontal física-psicológica, compuesto por 16 reactivos; Factor 2, violencia vertical desvalorización-control de profesores a estudiantes, compuesto por 11 reactivos; Factor 3, violencia vertical discriminación, compuesto por ocho reactivos; Factor 4, violencia vertical arbitrariedad, compuesto por ocho reactivos; Factor 5, violencia horizontal indiferencia-desprecio, 
con siete reactivos; Factor 6, violencia horizontal desaprobación-rechazo, con siete reactivos; Factor 7, violencia vertical abuso, con cuatro reactivos; Factor 8, violencia horizontal calumnia, con tres reactivos, que hacen un total de 64 reactivos. Lo anterior permite afirmar que se cubrió satisfactoriamente el objetivo general planteado.

Con relación a los tipos de violencia, se comprobó la existencia de algunas prácticas violentas verticales y horizontales, predominando la violencia entre pares estudiantes, sobre el binomio de violencia de estudiante-profesor; así también, la presente constituyó una posibilidad concreta para confirmar la hipótesis planteada, existen prácticas violentas horizontales entre estudiantes de educación pública superior. Factor 1, violencia horizontal física-psicológica, Factor 5, violencia horizontal indiferencia-desprecio, Factor 6, violencia horizontal desaprobación-rechazo, Factor 8, violencia horizontal.

En tercer lugar, sobre las prácticas violentas verticales, se confirma la hipótesis: existen prácticas violentas verticales entre estudiantes y profesores de educación pública superior con los resultados encontrados en el Factor 2, violencia vertical desvalorización-control de profesores a estudiantes, Factor 3, violencia vertical discriminación, Factor 4, violencia vertical arbitrariedad, Factor 7, violencia vertical abuso.

Las medias por sexo revelan que los varones emiten significativamente mayor violencia que las mujeres, lo que permite afirmar la vigencia de estereotipos culturales por género. Y que se ve reforzado por un contexto que constante y sistemáticamente somete a los jóvenes a cantidades descomunales de violencia como consumidores. Los registros de las medias por sexo podrán ser utilizados como punto de comparación para otras poblaciones a nivel nacional e internacional.

La EVIEES aporta la identificación de prácticas violentas entre estudiantes en términos de exclusión, violencia psicológica, sobretodo en dos diferentes modalidades, indiferencia y desvalorización, que en contraste con las prácticas de este orden de los estudiantes de educación 
básica y media superior, se distinguen por su sutileza, porque se mantienen en el orden de lo simbólico con aproximaciones acotadas que van de leves a moderadas en términos físicos. Sin embargo, en virtud de la apertura a nuevos mundos de vida por su joven adultez permanece la interrogante sobre las formas de violencia sexual, tales como acoso, abuso sexual, violación, disfrazadas de cortejo y seducción forzada.

Además, es necesario considerar el movimiento dialéctico del contexto que oscila entre libertad, hedonismo y riesgo, y en sus consecuencias incentiva pautas de comportamiento inseguras, conflictivas, e inestables para ambos géneros. Por otro lado, la dinámica social provoca procesos de capilarización de los diferentes grupos sociales, independientemente de su perfil sociopsicológico, lo que a su vez genera una exposición de pautas ajenas a las propias que pueden ser o no asimilables al punto de hacerlas propias. Lo anterior significa que, existe el peligro que las pautas violentas extremas en el orden de lo simbólico se propaguen a otros perfiles colectivos de menor edad, en otras palabras, la violencia sutil de los estudiantes de educación superior puede ser radiada a adolescentes y niños, a su vez la violencia concreta de los casos extremos registrados en la educación básica y media superior guarda la posibilidad de ser arrastrada hasta la educación superior (Abramovay, 2005).

Este patrón polimorfo de la violencia, demanda un monitoreo constante en los diferentes cortes que desde la psicología puedan concebirse, entre ellos, el desarrollo, los rasgos, las estructuras psíquicas, por mencionar algunos. Abordando el fenómeno a partir de diferentes actores sociales, como bien pueden ser los grupos indígenas, personas con discapacidad específica (diversidad funcional), estudiantes hetero-normativos y no hetero-normativos, entre otros. 


\section{Anexo 1. EVIEES (Delgadillo, Argüello, González, 2016)}

\section{Universidad Autónoma Del Estado De México}

Facultad De Ciencias De La Conducta

El cuestionario que se presenta a continuación es parte de una investigación que se lleva a cabo en la Facultad de Ciencias de la Conducta llamada: "Prácticas Violentas por Género en Estudiantes de Educación Pública Superior" de la Universidad Autónoma del Estado de México, te invitamos a participar, ten presente que no hay respuestas correctas o incorrectas, tu participación se mantendrá con carácter anónimo y confidencial ; lo que reportes será analizado estadísticamente para el diseño de una herramienta psicométrica.

DATOS GENERALES
EDAD:__ SEXO: Hombre ( ) Mujer ( ) GÉNERO:

INSTRUCCIONES: : A continuación se presenta un conjunto de afirmaciones, léelas con atención y responde si dichas situaciones se han presentado en tu escuela en la convivencia entre compañeros(as) y, entre profesores(as) y alumnos(as). Marca con una "X" la casilla según sea tu experiencia; donde: MF - Muy
Frecuente
F - Frecuentemente
AV - Algunas Veces
$\mathrm{CN}$ - Casi Nunca
$\mathrm{N}$ - Nunca

\begin{tabular}{|c|c|c|c|c|c|c|}
\hline & tre Compañeros(as) & $\mathrm{MF}$ & $\mathrm{F}$ & AV & $\mathrm{CN}$ & $\mathrm{N}$ \\
\hline 1 & Agreden a otros sin causa aparente. & & & & & \\
\hline 2 & Se golpean. & & & & & \\
\hline 3 & Se critican por su forma de ser. & & & & & \\
\hline 4 & Se llevan de manera tosca. & & & & & \\
\hline 5 & Se crean chismes divulgándolos entre ellos. & & & & & \\
\hline 6 & Se callan groseramente. & & & & & \\
\hline 7 & Se gritan. & & & & & \\
\hline 8 & Se dejan de hablar sin motivo aparente. & & & & & \\
\hline 9 & Utilizan el chantaje para beneficio personal. & & & & & \\
\hline 10 & Se burlan públicamente. & & & & & \\
\hline 11 & Se ignoran como una forma de agredirse. & & & & & \\
\hline 12 & Se desacreditan con chismes. & & & & & \\
\hline 13 & Se arrebatan las cosas. & & & & & \\
\hline 14 & Se hablan mal entre sí. & & & & & \\
\hline 15 & Hahlan mal a sus esnaldas sin razón & & & & & \\
\hline
\end{tabular}


Delgadillo, L.; Argüello, F.; González, L.

19 Se saludan de "bulto" (con golpes, empujones).

20 Se miran "feo".

21 Se dejan con "la palabra en la boca".

22 Se dicen palabras obscenas.

23 Esparcen rumores para desacreditar a un(a) compañero(a).

24 Se rechazan evitando sentarse junto a un compañero(a).

25 Se humillan.

26 Aplican la ley del hielo.

27 Cuando pasan junto al otro(a) se empujan sin motivo.

28 Se excluyen de trabajos por no caerse bien.

29 Se critican por la ropa que usan.

30 Se lanzan objetos.

31 Se insultan por ser de diferente clase social.

32 Se amenazan entre sí.

33 Se ofenden.

\section{Entre Profesores(as) y Alumnos(as)}

34 Muestran indiferencia a los alumnos(as) indisciplinados(as).

35 Deja una mayor carga de trabajo, superior a la indicada en el programa.

36 Condicionan la calificación a cambio de un aporte material.

37 Los guapos(as) son sus preferidos(as).

38 Abusan de su autoridad.

39 Relegan a los(as) estudiantes que no les simpatizan.

40 Dan menos valor al trabajo del estudiante discapacitado(a).

41 Instan al alumno(a) a realizar actividades para burlarse de ellos(as).

42 Intimidan al alumno(a) para que realice "algo" para ellos(as).

43 Dan órdenes abusando de su autoridad.

44 Cambian los lineamientos de la entrega de trabajos sin previo aviso.

45 Tienen a sus preferidos(as).

46 Favorecen a los que visten bien.

47 Son indiferentes con los alumnos(as) menos "listos"(as).

48 Rechazan a los(las) feos(as).

49 Rechazan al alumno(a) que es homosexual.

50 Presentan los trabajos de los alumnos(as) como propios. 


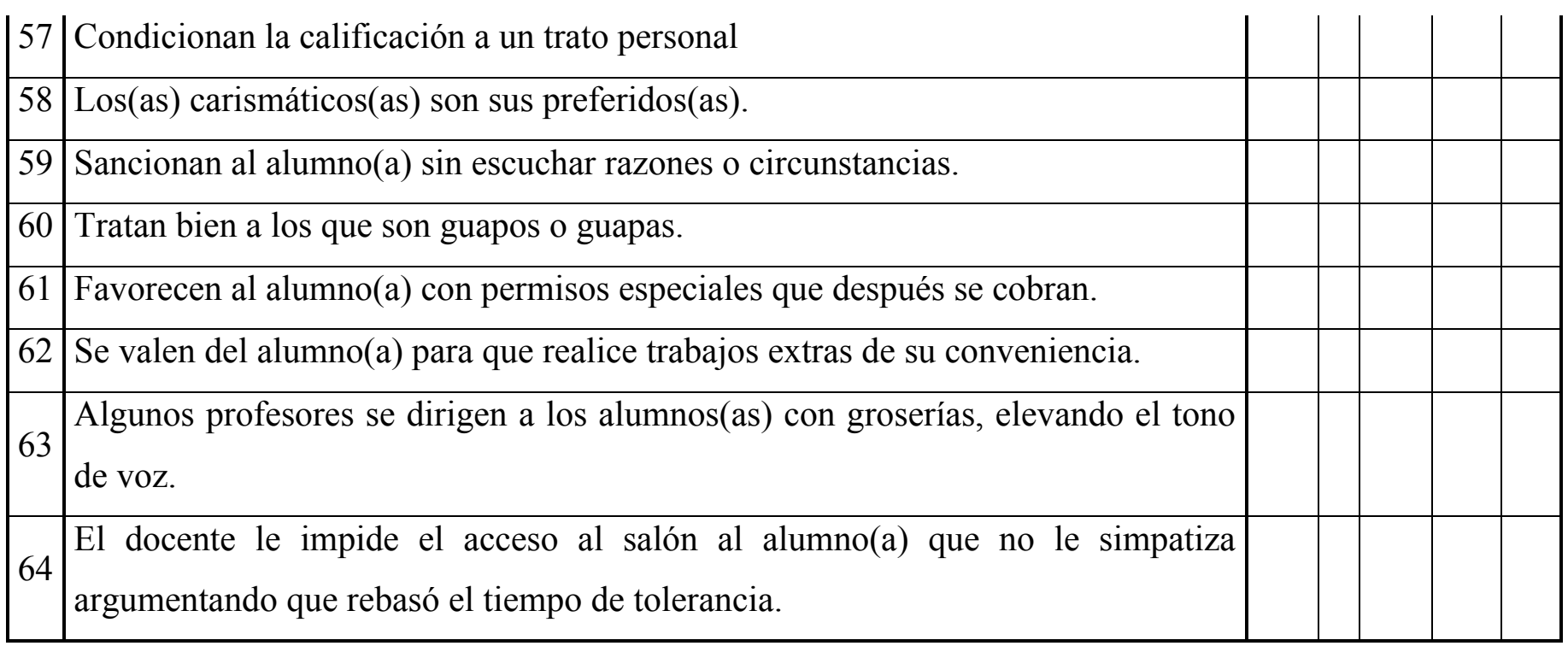

¡MUCHAS GRACIAS POR TU PARTICIPACIÓN! 


\section{Bibliografía}

Abramovay M. (2005). Victimización en las escuelas. Ambiente escolar, robos y agresiones físicas. Revista Mexicana de Investigación Educativa. 10, 833-864.

Bellido, E. (2010). Prospección al fenómeno del bullying y la violencia escolar. Revista de Estudios Policiales. 6, 11-28.

Berger, P. y Luckmann, T. (1978). La construcción social de la realidad. Buenos Aires: Amorrortu.

Cerezo, F. (2001). Variables de personalidad asociadas en la dinámica bullying en niños y niñas de 10 a 15 años. Anales de Psicología. Universidad de Murcia. Recuperado de www.redalyc.com.

Constitución Política de los Estados Unidos. DE: http://www.ordenjuridico.gob.mx/Constitucion/cn16.pdf, consultado el 28 de febrero 2017.

Del Barrio, C., Marín, E. Fernández, I., Hierro, L., Montero, I., Gutiérrez, H., Ochaita, E. (1999). Informe sobre la violencia escolar: el maltrato entre iguales en la educación secundaria obligatoria. España: Universidad Autónoma de Madrid.

Delgadillo, L., y Mercado, A. (2010). Violencia laboral, una realidad incómoda. Estudios de casos desde perspectiva de género. México: Universidad Autónoma del Estado de México. Delgadillo, L., Argüello, F., González, L., García, S. (2012). El bullying en la educación pública superior en México. Ciencia Tecnología e Innovación para el desarrollo de México. Año 4, PCTI 97. 
Del Rey, R., y Ortega, R. (2001). Programas para la prevención de la violencia escolar en España: la respuesta de las comunidades autónomas. Revista Interuniversitaria de Formación del Profesorado. 41, 133-145.

Del Rey, R., y Ortega, R. (2008). "Bullying en los países pobres: prevalencia y coexistencia con otras formas de violencia". International Journal of Psychology and Psychological Therapy. 8 (1), 39-50.

Debarbieux, E., Blaya, C. (2002). Violencia nas escolas Dez Abordagens Européias. UNESCO: Brasil.

Fisher, R. (2010). Bullying escolar nao Brasil. Relatório final. Brasil: Centro de Empreendedorismo Social e Administração em Terceiro Setor, Fundação Instituto de Administração.

Hernández, R., Fernández, C., y Baptista, P. (2003). Metodología de la investigación. $3^{\text {a }}$ Ed. México: Mc Graw Hill.

Krauskof, D. (2006). Estado del arte de los programas de prevención de violencia en ámbitos escolares. Organización Panamericana de la Salud 2006. Recuperado de http://www.paho.org/spanish/ad/fch/ca/arte.violencia.pdf

Letamendia Perez, R. (2002). El maltrato en los contextos escolares. Revista de Psicodidáctica. Universidad del País Vasco. 
Nansel, T., Craig, W., Overpeck, M., Saluja G., Ruan W., SImons-Morton B., Scheidt, P. (2004). Bullying behaviours among US youth: Prevalence and association with psychosocial adjustment. Journal of the American Medical Association. 285, 2094-2100.

Olaiz F., Rivera, D., Shamah, L., Rojas, R., Villalpando, H. (2006). Encuesta Nacional de Salud y Nutrición 2006. México: Instituto Nacional de Salud Pública.

Olweus, D. (1998). Conductas de acoso y amenaza entre escolares. España: Morata.

Olweus, D. (2003). Bullying/victim problems in school: basic facts and an effective intervention programme. En Einrasen, S., Hoel, H., Zapf, D., Cooper, C. (Eds). Bullying and emotional abuse in the workplace. 62-67. Londres: Taylor and Francis.

Olweus, D. (2006). Conductas de acoso y amenaza entre escolares. Perú: Alfaomega.

Ortega, R. (2005). Violencia escolar en Nicaragua. Un estudio descriptivo en escuelas de primaria. Revista Mexicana de Investigación Educativa. 10 (26), 787-804.

Ortega, R., Calmaestra, J., Mora, J., (2001). Violencia entre escolares. Conceptos y etiquetas verbales que definen el fenómeno del maltrato entre iguales. Revista Interuniversitaria de Formación del Profesorado. 41, 95-113.

Ortega, R., Calmaestra, J., Mora, J., (2008). Cyberbullying. International Journal of Psychology and Psychological Therapy. 8 (2), 183-192.

Ortega, R.; Del Rey, R.; Sánchez, V.; Ortega-Rivera, J.; Genebat, R. (2005). Violencia escolar en Nicaragua. Un estudio descriptivo en escalas de primaria Revista Mexicana de investigación Educativa Sección temática problemas de indisciplina y violencia en la escuela. 10 (26), 787-804. 
Ortega, R., y Del Rey, R. (2008). La violencia escolar. Estrategias de prevención. España: Grao.

Rodulfo, J. (2011). El bullying en Ciudad Juárez: Un análisis descriptivo del fenómeno. XI Congreso nacional de Investigación Educativa. 17. Convivencia, disciplina y violencia en las $\begin{array}{llll}\text { escuelas } & \text { (Ponencia). }\end{array}$ http://lab.iiiepe.net/congresonacional/docs/area_17/0316.pdf

Romans, M. (2000). De profesión educador (a) social. España: Paidós.

Ruiz, O. (1989). Metodología de la investigación cualitativa. Bilbao: Universidad de Deusto.

Schutz, A. (1993). El problema de la realidad social. Buenos Aires: Amorrortu editores.

SEP, UNICEF México (2009). Informe Nacional Sobre Violencia de Género en la Educación Básica en México 2009. México: Secretaria de Educación Pública.

Thorndike, R. (1989). Psicometría aplicada. México: Limusa.

Valadez-Figueroa I. (2008). Violencia escolar: maltrato entre iguales en escuelas secundarias de la zona metropolitana de Guadalajara. Informe de estudio. México: Colección Salud Materno Infantil.

Velázquez, L. (2008). Violencia en la escuela: convivencia y experiencias de riesgo en alumnos de ocho escuelas secundarias de la ciudad de Toluca, México. Primer congreso sobre paz, democracia y Desarrollo. Mesa II Comunicación, Educación y Cultura de Paz. Recuperado de http://politicas.uaemex.mx/comuniv/congreso/MESA2/MESA2B/M2BP1.pdf 
Construcción de la EVIEES: Prácticas violentas por género en Educación Superior 